\title{
Entrainment in Cumulus Clouds: What Resolution is Cloud-Resolving?
}

\author{
George C. Craig and Andreas Dörnbrack \\ Institut für Physik der Atmosphäre, DLR Oberpfaffenhofen, Wessling, Germany
}

(Manuscript received 5 September 2007, in final form 2 April 2008)

\begin{abstract}
Systematic numerical experiments were conducted to determine the spatial resolution required to resolve a moist thermal show convergence at a scale proportional to the smaller of the initial thermal diameter $D_{0}$ and a buoyancy length scale $L_{\text {buoy }}$. The buoyancy length scale $L_{\text {buoy }}=\Delta T_{0} / \Delta \Gamma\left(\Delta T_{0}\right.$ is the initial buoyancy excess of the thermal and $\Delta \Gamma$ is the ambient stratification) describes the maximum vertical displacement that can be induced against the stratification in the environment by buoyancy-driven pressure perturbations in the cloud and, thus, the maximum scale of eddies that cross the cloud boundary. For typical atmospheric conditions in which the cloud size $D_{0}$ is larger than $L_{\text {buoy }}$, numerical simulations of the mixing processes in cumulus clouds must resolve $L_{\text {buoy. }}$.
\end{abstract}

\section{Introduction}

In atmospheric modeling, one of the most difficult processes to simulate is cumulus convection. Convection is generally parameterized in weather and climate models, but there is increasing interest in performing simulations with horizontal resolutions in the $1-3-\mathrm{km}$ range without a cumulus parameterization scheme. Comparison studies have shown conclusively that such models perform better than single column models with a convection scheme (Bechtold et al. 2000; Guichard et al. 2004). Although they are sometimes referred to as "cloud resolving," numerical models with a $1-\mathrm{km}$ grid size clearly do not have sufficient resolution to accurately represent the complex turbulent flow that makes up a cumulus cloud. But what spacing is necessary to accurately simulate the transport, entrainment, and detrainment processes in such clouds?

The properties of the moist turbulence in cumulus clouds are only partially understood, particularly at the cloud boundary where there is entrainment of environmental air and detrainment of cloud air into the environment (Bretherton 1997). Additionally, the wide range of scales involved in convection makes it computationally very costly to demonstrate convergence by

Corresponding author address: Andreas Dörnbrack, Institut für Physik der Atmosphäre, DLR Oberpfaffenhofen, D-82230 Wessling, Germany.

E-mail: andreas.doernbrack@dlr.de brute force, although simulations with a resolution of $100 \mathrm{~m}$ or better have been performed (see Bryan et al. 2003; Stiller and Craig 2001; Khairoutdinov and Randall 2006). For many turbulent flows, laboratory data provide a reference (see Andrejczuk et al. 2004, 2006), but no suitable laboratory analog has been discovered for the phase change of condensing water in cumulus clouds.

Kilometer-scale cloud models, sometimes referred to as cloud-resolving or "cloud permitting" models, typically use a subgrid turbulence parameterization designed for large-eddy simulation (LES), in which it is assumed that the dominant scales of the turbulence are resolved and the overall characteristics of the simulation are independent of the details of the subgrid-scale closure. But for cumulus convection it is not known what the dominant turbulent scale is, or whether one exists at all. Thus, it is not certain that a large-eddy approximation is valid, or at what resolution. Naively applying a turbulence parameterization designed for LES in a numerical model with a grid length comparable to the dominant scale of the turbulence leads to errors associated with the omission of some of the turbulent energy production terms (Wyngaard 2004). The aim of this paper is to identify a possible dominant scale for turbulence in cumulus clouds and to provide some indication of convergence of bulk cloud properties if this scale is resolved.

As a starting point, we consider a problem that is well understood: the buoyant thermal (Emanuel 1994; 


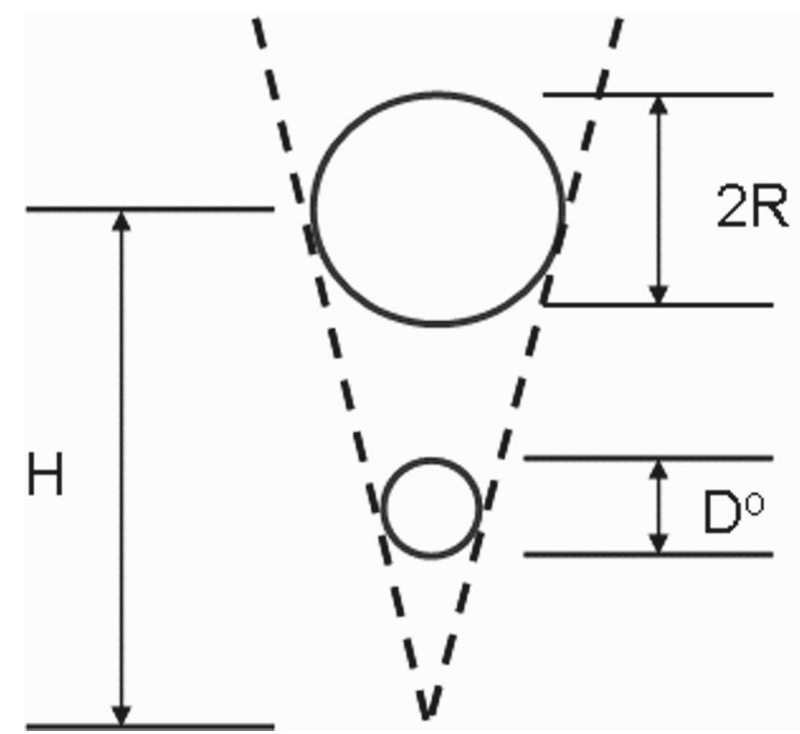

FIG. 1. Schematic of a buoyant thermal rising in a neutrally stratified atmosphere.

Houze 1993). The basic version of this problem considers a warm bubble of air rising in a neutrally stratified atmosphere, shown schematically in Fig. 1. When the bubble has risen a distance $H$ that is large in comparison with the initial bubble diameter $D_{0}$ (in laboratory experiments a distance of about three bubble diameters is sufficient), the turbulence is fully developed and the initial bubble shape is forgotten. The subsequent evolution of the thermal is self-similar, growing linearly with height. The evolution is determined by a single dimensional parameter, the initial total buoyancy $Q$ :

$$
Q=\int g \frac{T^{\prime}}{T_{\text {ref }}} d V=g \frac{\Delta T_{0}}{T_{\text {ref }}} V_{0},
$$

where $g$ is the gravitational acceleration, $\Delta T_{0}$ is the initial temperature excess, $V_{0}$ is the volume of the bubble, and $T_{\text {ref }}$ is a reference temperature. Dimensional analysis gives the following solutions for the time evolution of the radius $R$, height $H$, and volume $V$ of the thermal:

$$
\begin{aligned}
& R=a Q^{1 / 4} t^{1 / 2}, \\
& H=b Q^{1 / 4} t^{1 / 2}, \\
& V=c Q^{3 / 4} t^{3 / 2},
\end{aligned}
$$

where $a, b$, and $c$ are universal constants. In particular, the solutions show the linear relation between the radius and height of the thermal: $R=(a / b) H$, where the ratio $a / b$ is found in laboratory experiments to be about 0.1 (Morton et al. 1956). Because all length scales in this problem are proportional to the height (or radius), this also specifies the dominant scale of the turbulence.
Thus, one would expect to find a large-eddy regime in which turbulence on scales smaller than the overall size of the thermal could be parameterized. If one has a sufficient number of grid points across the thermal at its initial smallest $D_{0}$, an accurate simulation should be possible.

The buoyant thermal has also been treated for a stratified environment. In this case, a new length scale enters in the problem: the buoyancy height $L_{\text {buoy }}=$ $\Delta T_{0} / \Delta \Gamma$, where $\Delta \Gamma=d T / d z+g / c_{P}$ denotes the stratification, with $c_{P}$ the specific heat at constant pressure. If $L_{\text {buoy }}$ is much larger than the bubble size $D_{0}$, the turbulence in the thermal will be little affected by the stratification, and the buoyancy length scale determines the total height that the plume would rise in the absence of entrainment. Consequently, the thermal will remain self-similar, retaining the vortex ring structure characteristic of a plume in a neutrally stratified environment, and the rate of increase of the thermal's size (the rate of entrainment of environmental fluid) will still be proportional to height (Morton et al. 1956). This "entrainment hypothesis" is also applied in the plume models used to calculate the vertical profile of mass flux in many cumulus parameterizations. For a thermal in a stratified environment, the required resolution thus remains $D_{0}$, as long as $L_{\text {buoy }}$ is large enough. However, for a cumulus cloud, $D_{0}$ is typically $1 \mathrm{~km}$ or more, whereas $L_{\text {buoy }}$ is about $333 \mathrm{~m}$ (for $\Delta T_{0} \sim 1 \mathrm{~K}, \Delta \Gamma \sim 3$ $\mathrm{K} \mathrm{km}^{-1}$ ). In this case, $L_{\text {buoy }}$ is not large in comparison to $D_{0}$ and the entrainment hypothesis is not justified.

A systematic attempt to determine the resolution requirement for moist convection was made by Bryan et al. (2003). In simulations of a squall line with resolutions from $1 \mathrm{~km}$ to $125 \mathrm{~m}$, evidence was found for an inertial subrange in the 250- and 125-m-resolution experiments. At $125-\mathrm{m}$ resolution, the ratio of subgrid kinetic energy to total kinetic energy rarely exceeded $10 \%$. This provides some indication that an LES assumption may be valid, although properties such as maximum vertical velocity varied irregularly with resolution. A motivation for their study was the observation that simulations of the dry convective boundary layer show convergence with a grid spacing that is a factor of 100 smaller than the dominant scale of the eddies (equal to the boundary layer depth). For moist convection, if the size of the clouds is typically about $10 \mathrm{~km}$, a grid spacing of $100 \mathrm{~m}$ would then be required. However, this argument ignores the potential effect of the thermal stratification on the turbulence, which the scaling argument above suggests would be important.

An attempt to construct a theory valid for the parameter regime found in moist convection was made by Stiller and Craig (2001), who considered the problem of 




\section{Dry thermal}



\section{Moist thermal}

FIG. 2. Relevant mixing scales for the entrainment of a buoyant thermal in a dry and moist environment.

a warm, saturated bubble rising in a moist neutral environment. This situation is analogous to the basic dry thermal in the sense that in the absence of mixing, the bubble would continue to rise, always with the same buoyancy excess. Here, however, the environment is stratified, and it is the released latent heat of condensation that allows the moist thermal to remain buoyant. As noted previously, if $L_{\text {buoy }}$, the length scale associated with the stratification, is large compared to the size of the bubble $D_{0}$, the largest eddies are on the scale of the bubble itself, as in the dry case (Fig. 2, top). However, if $L_{\text {buoy }}$ is smaller than the bubble size $D_{0}$, the buoyancy of the bubble does not provide enough force to move the surrounding air against the stratification farther than the distance $L_{\text {buoy }}$. The turbulent eddies that cross the cloud boundary are thus limited in scale to $L_{\text {buoy }}$ (Fig. 2, bottom). This then represents the dominant scale of the turbulence associated with entrainment and constrains the spatial resolution required to accurately simulate the evolution of the moist thermal.

The central question of this paper is: Do simulations of a moist thermal converge for grid sizes smaller than $D_{0}$ or smaller than $L_{\text {buoy }}$ ? Numerical simulations of a moist thermal are presented using a fluid solver described in section 2 and an experimental configuration described in section 3. Suitable diagnostics to deter- mine convergence without impractical computational requirements are discussed in section 4 . A set of experiments in which the ratio of $D_{0} / L_{\text {buoy }}$ is varied and the resolution required for convergence is determined from sets of runs with different resolution is then described in section 5 . The conclusions are given in section 6.

\section{Numerical model}

Our numerical experiments were performed with the nonhydrostatic, anelastic model EULAG. A comprehensive description of the model and its capabilities can be found in Smolarkiewicz et al. (2001), Grabowski and Smolarkiewicz (2002), and Smolarkiewicz and Prusa (2005). The prognostic equations are written compactly in conservation law form:

$$
\frac{\partial \bar{\rho} \Psi}{\partial t}+\boldsymbol{\nabla} \cdot(\bar{\rho} \mathbf{v} \Psi)=\bar{\rho} R^{\Psi},
$$

where $\bar{\rho}$ is the anelastic density, $\nabla:=(\partial / \partial x, \partial / \partial y, \partial / \partial z), \Psi$ symbolizes the velocity components $u, v$, and $w$, the potential temperature $\Theta$, or the moist variables: here, the water vapor mixing ratio $q_{v}$, the cloud condensed mixing ratio $q_{c}$, and the rainwater mixing ratio $q_{r} ; R^{\Psi}$ symbolizes the associated source and sink terms. The governing equations (5) are solved by means of finite difference approximations using a second-order accurate nonoscillatory forward-in-time (NFT) approach (Smolarkiewicz and Margolin 1997). On a discrete mesh, the NFT approximation of (1) is written as

$$
\psi_{i}^{n+1}=\operatorname{LE}_{i}(\tilde{\psi})+\left.0.5 \Delta t R^{\psi}\right|_{i} ^{n+1},
$$

where $\psi_{i}^{n+1}$ denotes the solution at the grid point $\left(t^{n+1}\right.$, $\left.\mathbf{x}_{i}\right), \tilde{\psi}=\psi^{n}+0.5 \Delta t R^{n}$, and LE denotes the NFT transport operator. In the Eulerian scheme, used exclusively in this paper, LE integrates the homogeneous transport equation (1); that is, LE advects $\tilde{\psi}$ using a fully secondorder-accurate flux-form scheme (MPDATA; for a review, see Smolarkiewicz and Margolin 1998). Unlike the implicit formulation of the dynamical core of the model (see Dörnbrack et al. 2005), the moist variables are treated explicitly to accommodate a broad range of temporal scales [for further details of the microphysical scheme, see Grabowski and Smolarkiewicz (2002) and references therein]. As a default, all simulations were performed with the implicit large-eddy simulation (ILES) approach, in which the required subgrid-scale dissipation is provided by the truncation terms of the NFT schemes (cf. Domaradzki et al. 2003 and references therein). 


\section{Experimental setup}

The design of the control experiment is similar to that of Stiller and Craig (2001), but without the simplifications to the thermodynamic relations or microphysical parameterization that were introduced there to facilitate the mathematical analysis. The ascent of a warm, saturated bubble of air is simulated. The atmosphere is initially at rest, with a moist adiabatic lapse rate and a fixed saturation deficit in the humidity field.

The background states are prescribed as follows: $\bar{\rho}(z)=p_{m} /\left(R_{d} T_{m}\right)$ and $\bar{\Theta}(z)=T_{m}\left(p_{m} / p_{00}\right)^{-\left(R_{d} / c_{P}\right)}$, with $p_{m}(z)=p_{00}\left(1-R_{d} z / c_{P} H_{S}\right)^{\left(c_{P} / R\right)}$ as the background pressure profile; $T_{m}(z)$ is estimated iteratively as described below. Here, $R_{d}$ denotes the gas constant for dry air, $p_{00}=1000 \mathrm{hPa}$ is the surface pressure, and $H_{S}=8500 \mathrm{~m}$ is the scale height. The moist adiabatic temperature $T_{m}(z)$ is determined in such a way that the amount of water substance $\Delta q_{v}=C_{d} \Delta t$ subject to phase changes is evaluated by solving the transcendental equation

$$
q_{v}^{*}-\Delta q_{v}=q_{v s}\left(\Theta^{*}+\frac{L_{v} \Theta_{e}}{c_{P} T_{m}} \Delta q_{v}\right),
$$

starting from $z=0$ with $T_{m}(0)=T_{00}=287 \mathrm{~K}$ as the ground-level temperature (see section 3a in Grabowski and Smolarkiewicz 1990). The environmental states for the velocity components are set to zero $\left(\mathbf{u}_{e}=0\right)$, the environment potential temperature is equal to the background state $\Theta_{e}=\bar{\Theta}$, and the ambient water vapor mixing ratio is described as $q_{v e}=\max \left(q_{v s}-0.3 \mathrm{~g} \mathrm{~kg}^{-1}\right.$, $\left.0.05 q_{u s}\right)$; that is, the relative humidity decreases from about $97 \%$ at the ground to $5 \%$ at an altitude of $\sim 8 \mathrm{~km}$.

The spherical bubble is prescribed by the diameter $D_{0}$, the temperature excess $\Delta T_{0}$, and a relative humidity $(\mathrm{RH})=100 \%$. To accommodate a smooth transition to a subsaturated environment, the initial potential temperature anomaly $\Theta^{b}(r)$ as function of radius was calculated by

$$
\Theta^{b}=\left\{\begin{array}{lll}
\Delta T_{0} \cos ^{2}\left(\frac{3 \pi r}{5 D_{0}}\right) & \text { for } & r<5 D_{0} / 6 \\
0 & \text { elsewhere. }
\end{array}\right.
$$

Inside the bubble an initial water vapor anomaly $q_{v}^{b}$ is added in a similar way as $\Theta^{b}$, so that the bubble is close to saturation:

$$
q_{v}^{b}=\left\{\begin{array}{lll}
q_{u s}-q_{v e} & \text { for } & r<D_{0} / 3 \\
\left(q_{u s}-q_{v e}\right) \cos ^{2}\left[\frac{\pi}{D_{0}}\left(r-\frac{D_{0}}{3}\right)\right] & \text { for } & D_{0} / 3<r<5 D_{0} / 6 \\
0 & \text { elsewhere. }
\end{array}\right.
$$

The initial height of the bubble is set to $3000 \mathrm{~m}$; in the horizontal direction, the bubble is located in the middle of the computational domain. For the control experiment, the initial bubble size was $D_{0}=1000 \mathrm{~m}$ and the temperature excess was $\Delta T_{0}=1.0 \mathrm{~K}$, and simulations were conducted with six different grid lengths $\Delta x$ ranging from 50 to $280 \mathrm{~m}$ to determine where convergence was achieved. Additional experiments were then carried out with larger and smaller values of $D_{0}$ and $\Delta T_{0}$.

The three-dimensional computational domain has approximate dimensions of $18 \mathrm{~km} \times 18 \mathrm{~km}$ horizontally and $15 \mathrm{~km}$ in height (with the exception of one set of simulations described below). The exact dimensions of the domain depend on the spatial resolution (always uniform; $\Delta x=\Delta y=\Delta z$ ) and the number of processors used for the horizontal domain decomposition. To minimize computational costs it is desirable to make the domain as small as possible, but if the domain is too small, the mean stratification will change substantially during the simulation because the subsidence that com- pensates for the rising cloud will be confined to an artificially small region. The values used here were selected after some experimentation. For the simulations with $D_{0}=500 \mathrm{~m}$, a horizontal domain size of $11 \mathrm{~km} \times$ $11 \mathrm{~km}$ was used to reduce computational costs. This is justified because a smaller bubble size implies less vertical mass transport and, correspondingly, less area required for subsidence. This choice was confirmed by repeating one of the simulations in the original, larger domain, verifying that the results were essentially identical. A Rayleigh sponge layer, which attempts to reduce spurious reflections of vertically propagating waves, was placed in the upper $5 \mathrm{~km}$ of the domain. The thermodynamic variables were relaxed toward the environmental state at the lateral boundaries in a $2-\mathrm{km}$ wide band. The integration time is $60 \mathrm{~min}$. At the initial time, a random nonsymmetric noise of small amplitude is added to the initial vertical velocity distribution to break the symmetry that leads to strong unrealistic instabilities in the middle of the domain while the bubble is evolving. 
(a)

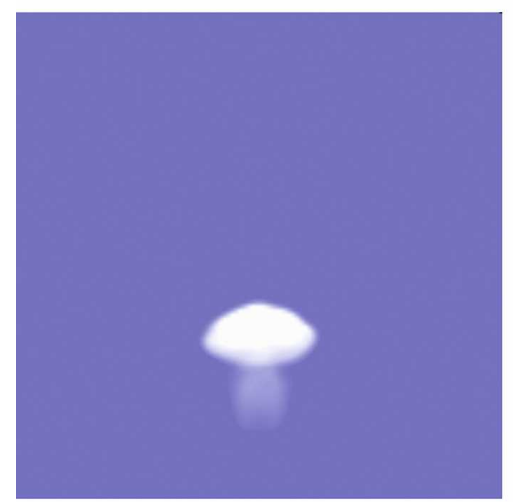

(d)

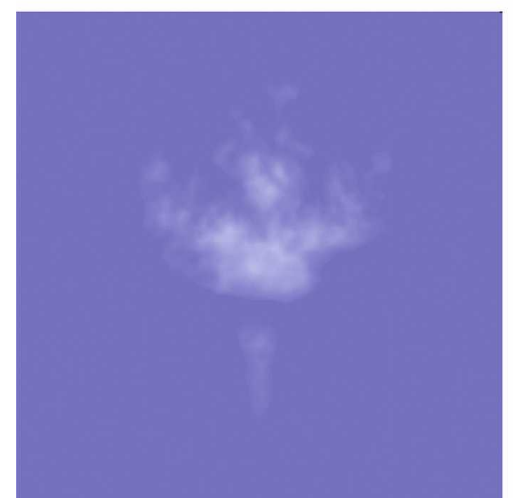

(b)

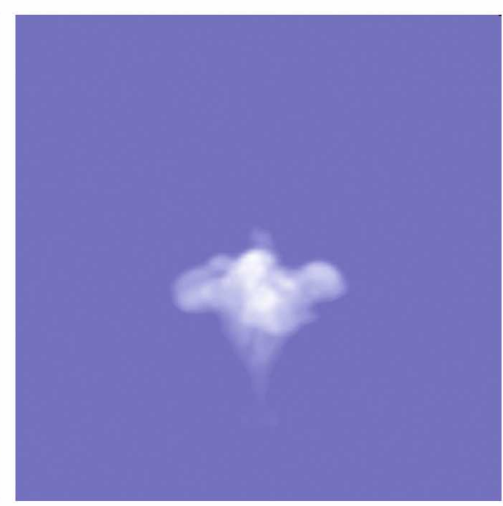

(e)

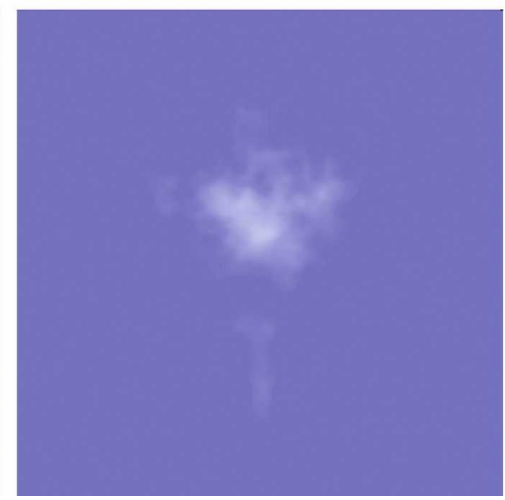

(c)

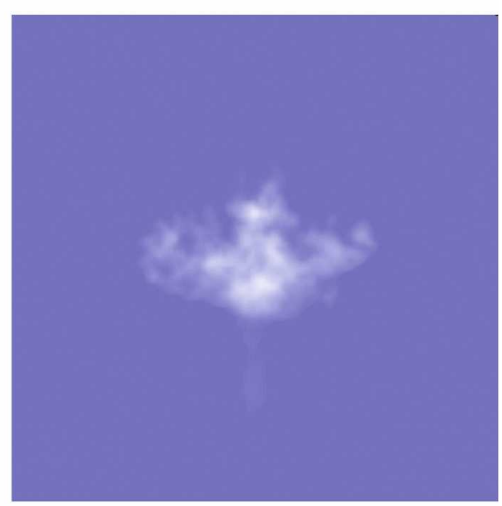

(f)

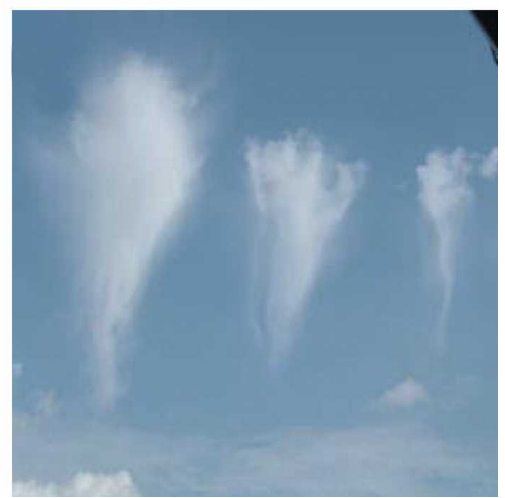

FIG. 3. (a)-(e) Renderings of the temporal evolution of the cloud water every 12 min starting at (a) $t=12$ min and ending at (e) $t=60 \mathrm{~min} ; \Delta T_{0}=1 \mathrm{~K}$ and $\Delta x=55 \mathrm{~m}$. (f) Photograph taken by J. Reveley at 1607 UTC 6 Jun 2004 in Reading, United Kingdom; see Reveley (2005).

\section{Control simulation and diagnostics of convergence}

Figure 3 shows renderings of the thermal with an initial temperature excess of $\Delta T_{0}=1 \mathrm{~K}$ and $\Delta x=55 \mathrm{~m}$ at 12-min intervals through the first hour. The images are created using a radiative transfer calculation to give a visual impression of the cloud evolution. The initially spherical bubble deforms into a mushroom-shaped cloud (Fig. 3a) that breaks up as the turbulence becomes fully developed (Figs. 3b-d). By the end of the simulation, the cloud is dissipating (Fig. 3e). The early appearance of the simulated cloud is rather unrealistic, with its smooth boundaries showing a lack of turbulent mixing with the environment. This leads to a rising cloud base because cloud air is not being detrained early in the evolution. Carpenter et al. (1998) have shown that this problem can be avoided by ensuring that the simulation has a realistic distribution of boundary layer turbulence in the initial conditions. However, for this study we keep the simple initial conditions to ensure that the runs are easily reproducible. In contrast, the later stages of the evolution of the simulated clouds appear strikingly realistic, as can be seen by comparison with Fig. 3f, a photograph of an observed cumulus humilis (Reveley 2005).

A quantitative view of the cloud evolution is given in Figs. 4a-c, in which expressions for the height $H$, volume $V$, and mean radius $R$ of the cloud are plotted as functions of time (see appendix). The particular functional dependences of these quantities are based on Eqs. (2)-(4), so the plots would be straight lines if the solutions for the dry thermal were valid. In each panel, two simulations that were started from slightly different initial conditions are shown. There is considerable variability between the two simulations, with different realizations of the turbulence leading to quite different rates of mixing and to correspondingly different cloud sizes and heights at any given time.

The large variability seen in Figs. $4 \mathrm{a}-\mathrm{c}$ has the potential to make any attempt to demonstrate convergence very expensive because one would need to average over a sufficiently large ensemble of already costly simulations to remove this variability. An alternative is to 

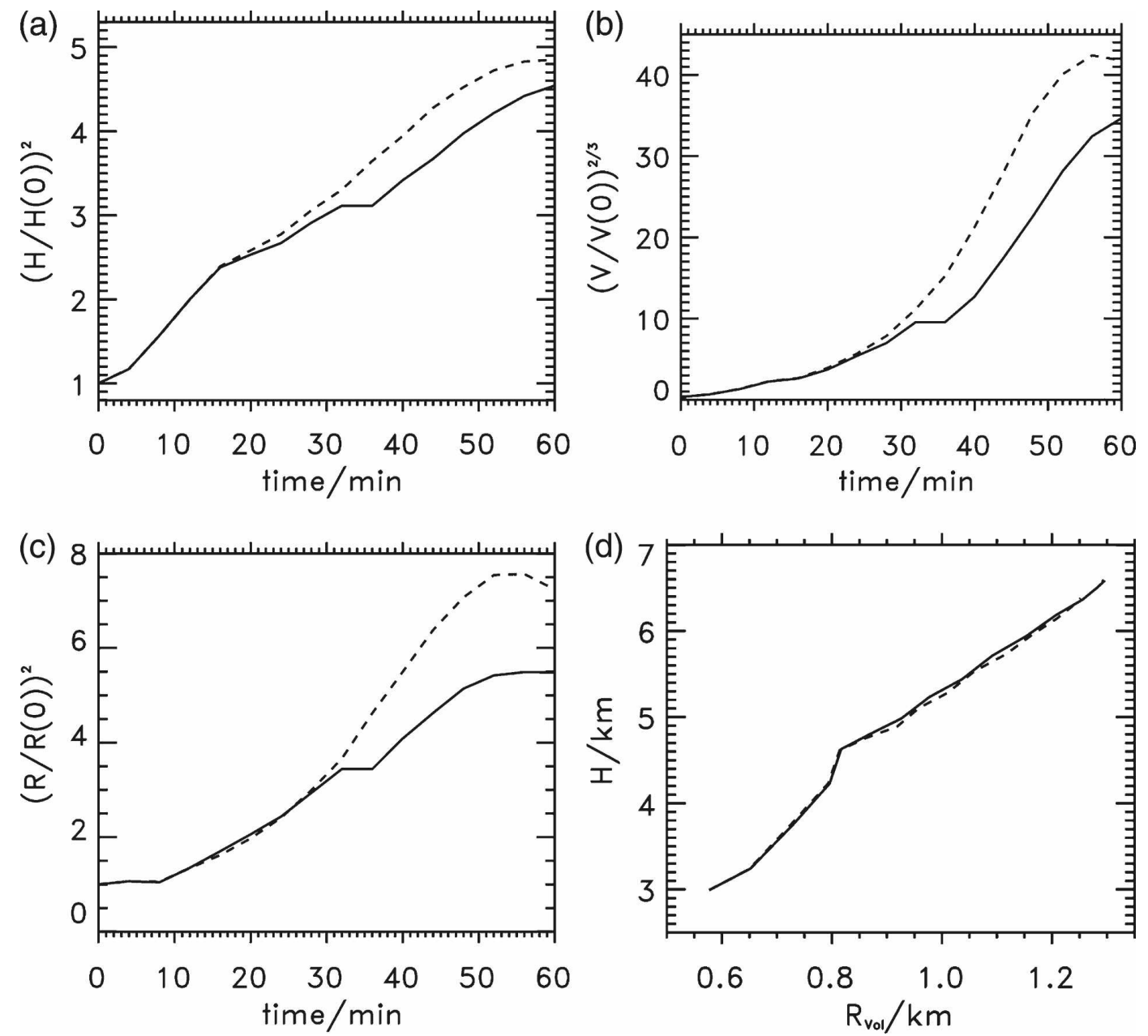

FIG. 4. Temporal evolution of the (a) normalized height, (b) volume (b), and (c) radius of the simulated thermal according to the scaling relationships of Eqs. (2)-(4). The different lines mark two distinct simulations for $\Delta x=70$ $\mathrm{m}$ and $\Delta T_{0}=1 \mathrm{~K}$ perturbed with slightly different initial conditions. (d) An alternative scaling using the averaged radius $R_{\mathrm{Vol}}=(3 V / 4 \pi)^{(1 / 3)}$.

examine measures of the flow that respond to integrated properties of the turbulence and are thus less sensitive to details of the particular numerical solution at a given moment. In particular, it appears from Figs. $4 a-c$ that the development of the thermal in one of the simulations is delayed in time relative to the other, whereas Eqs. (2) and (3) suggest that the height and radius of the thermal should be linearly related, independent of time.

Although the linear relationship was predicted for a dry thermal, Fig. 4d demonstrates that it also holds very well for the moist thermal once the turbulence is fully developed. The transition from the initial, more laminar evolution to the turbulent phase at $R_{\mathrm{Vol}} \approx 0.8 \mathrm{~km}$ is clearly seen. Importantly, the variability between the two simulations is almost completely removed. Therefore, we propose to use the radius-height plot to assess convergence. Convergence is achieved when the differences between simulations with differing resolutions are comparable to the difference between the two runs with perturbed initial conditions, as seen in Fig. 4d.

\section{Scale dependence of convergence}

Figure $5 \mathrm{~b}$ shows the height-radius plot for the control simulation $\left(\Delta T_{0}=1 \mathrm{~K}, D_{0}=1000 \mathrm{~m}\right)$ for six model runs with different spatial resolutions with $\Delta x=280$, $140,80,70,60$, and $50 \mathrm{~m}$, respectively. At the coarsest resolution the curve is quite irregular. At $80-\mathrm{m}$ resolution, the qualitative pattern of an initial laminar phase in which the radius increases slowly with height, followed by the turbulent phase with a linear radiusheight relationship, is present, but the slope differs quantitatively from the higher-resolution runs. The 50-, 

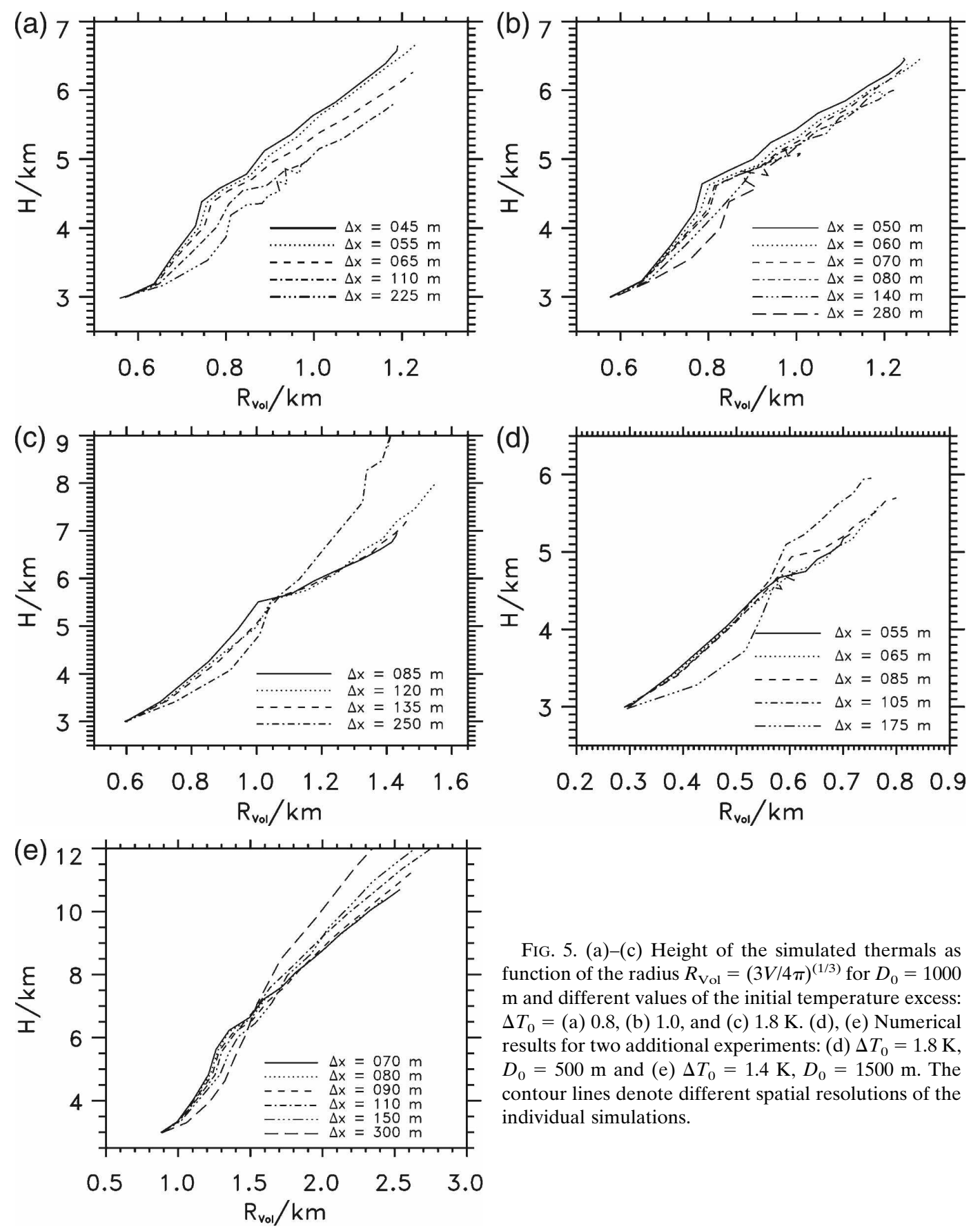

FIG. 5. (a)-(c) Height of the simulated thermals as function of the radius $R_{\mathrm{Vol}}=(3 V / 4 \pi)^{(1 / 3)}$ for $D_{0}=1000$ $\mathrm{m}$ and different values of the initial temperature excess: $\Delta T_{0}=$ (a) 0.8 , (b) 1.0 , and (c) $1.8 \mathrm{~K}$. (d), (e) Numerical results for two additional experiments: (d) $\Delta T_{0}=1.8 \mathrm{~K}$, $D_{0}=500 \mathrm{~m}$ and (e) $\Delta T_{0}=1.4 \mathrm{~K}, D_{0}=1500 \mathrm{~m}$. The contour lines denote different spatial resolutions of the individual simulations.

60-, and 70-m simulations produce very similar curves, with only a small offset due to a systematic decrease in entrainment in the laminar phase with increasing resolution. The similarity between these three simulations satisfies the convergence criterion defined in the previous section.

The required resolution $\Delta x=70 \mathrm{~m}$ for convergence in the control simulation is small compared to both candidate length scales $D_{0}$ and $L_{\text {buoy. }}$. To investigate the relevance of the two scales, sensitivity experiments will now be considered in which $L_{\text {buoy }}$ and $D_{0}$ are varied. Changes to $L_{\text {buoy }}$ will be made by varying the initial buoyancy of the bubble $\Delta T_{0}$ while the environmental stratification, determined by the moist adiabatic lapse rate, remains unmodified. In general, two regimes can be expected. First, $L_{\text {buoy }} \ll D_{0}$, in which turbulence on the scale of the bubble is suppressed by the stratification and $L_{\text {buoy }}$ controls convergence. Second, if $L_{\text {buoy }} \geq$ 
TABLE 1. Parameters for the control and four sensitivity experiments.

\begin{tabular}{cccc}
\hline \hline Experiment & $L_{\text {buoy }} / D_{0}$ & $D_{0}(\mathrm{~m})$ & $\Delta T_{0}\left(L_{\text {buoy }}\right)(\mathrm{K} ; \mathrm{m})$ \\
\hline Control & 0.33 & 1000 & $1.0(333)$ \\
1 & 0.27 & 1000 & $0.8(600)$ \\
2 & 0.60 & 1000 & $1.8(600)$ \\
3 & 1.20 & 500 & $1.8(600)$ \\
4 & 0.31 & 1500 & $1.4(470)$ \\
\hline
\end{tabular}

$D_{0}$ eddies can exist on the scale of the bubble diameter, $D_{0}$ and this scale will determine convergence.

A potential complication in the interpretation of these experiments is that the two length scales may change during the course of the simulation. However, these changes are not expected to alter the relative importance of the two length scales. On the one hand, the size of the thermal increases with time, so the initial bubble diameter $D_{0}$ represents the smallest value that will occur. If the size of the bubble is the important scale, then a grid size small enough to resolve the initial $D_{0}$ will suffice throughout the simulation. On the other hand, $L_{\text {buoy }}$ is affected by two opposing tendencies. The buoyancy excess will decrease as the thermal is diluted by entrainment, but the stratification of the environment will also become weaker as the thermal ascends (recall that the environmental temperature profile follows a moist adiabat). Eventually, the buoyancy of the bubble tends to zero and the thermal stops rising and dissipates, implying that $L_{\text {buoy }}$ has also decreased to zero. However, for much of the simulation time the two opposing effects keep $L_{\text {buoy }}$ relatively constant, implying that a resolution adequate for the initial $L_{\text {buoy }}$ should suffice until the final dissipating phase.

In addition to the control experiment, the resolution required for convergence will be identified in four experiments with modified initial conditions. The parameters for the control and four sensitivity experiments are summarized in Table 1.

In the first two sensitivity experiments, $D_{0}$ is kept at $1000 \mathrm{~m}$ while $L_{\text {buoy }}$ is decreased or increased by changing $\Delta T_{0}$. If convergence is controlled by $L_{\text {buoy, }}$, the required resolution will change in proportion with $\Delta T_{0}$, whereas if $D_{0}$ is the relevant scale, convergence should be achieved at the same resolution as in the control experiment. The values of $L_{\text {buoy }}$ in these three experiments are all smaller than $D_{0}$. Therefore, a third sensitivity experiment with $D_{0}<L_{\text {buoy }}$ is considered. Finally, we perform a fourth sensitivity experiment in which both $L_{\text {buoy }}$ and $D_{0}$ are increased. For this experiment, the ratio of the two length scales is very similar to the control experiment, but it is obtained with different parameter values, providing a test of similarity. In other words, the numerical results of this experiment should behave exactly like the control run if only the ratio of the two length scales is important in the problem.

Figure 5a shows the height-radius relation for the first sensitivity experiment $\left(\Delta T_{0}=0.8 \mathrm{~K}, D_{0}=1000 \mathrm{~m}\right)$ with spatial resolutions of $225,110,65,55$, and $45 \mathrm{~m}$. The integral behavior of the thermal's evolution is qualitatively similar to the control experiment: for $\Delta x=$ 110 and $65 \mathrm{~m}$ the slope is too shallow, but the 45- and 55-m-resolution simulations produce similar curves, satisfying the convergence criterion.

Figure $5 \mathrm{c}$ shows the height-radius relation for the second sensitivity experiment $\left(\Delta T_{0}=1.8 \mathrm{~K}, D_{0}=1000\right.$ $\mathrm{m})$ with resolutions of $250,135,120$, and $85 \mathrm{~m}$. The form of the curves here is somewhat different than those in the previous two experiments, as will be discussed in more detail below, but it is clear that the three higherresolution simulations are sufficiently similar during the turbulent phase to satisfy the convergence criterion at 135-m resolution.

The results of the third sensitivity experiment $\left(\Delta T_{0}=\right.$ $1.8 \mathrm{~K}, D_{0}=500 \mathrm{~m}$ ) are shown in Fig. $5 \mathrm{~d}$ for resolutions of $175,105,85,65$, and $55 \mathrm{~m}$, respectively. Here, it can be seen that the convergence criterion is satisfied at 65-m resolution. The qualitative behavior of the curves is similar to the second experiment. In contrast to the control experiment, the underresolved thermals in the turbulent phase have excessively small volumes at a given height (i.e., the curves lie to the left of those of the resolved runs).

Finally, Fig. 5e shows that the fourth experiment $\left(\Delta T_{0}=1.4 \mathrm{~K}, D_{0}=1500 \mathrm{~m}\right)$ behaves qualitatively similarly to the control experiment. Simulations with resolutions of $300,150,110,90,80$, and $70 \mathrm{~m}$ show radiusheight curves nearly identical to those for the 80- and 70-m runs, and even the 90-m-simulation curve appears to be close enough to satisfy the criterion defined in section 4 .

To understand the different qualitative behavior in the second and third sensitivity experiments, recall that if $L_{\text {buoy }}$ is larger than $D_{0}$, then the turbulence on the scale of the thermal will not be affected by the stratification and $L_{\text {buoy }}$ becomes irrelevant. In this case, the turbulent plume will exhibit a vortex ring structure similar to that which would occur in a neutrally stratified environment. For the second and third sensitivity experiments, with the largest values of $L_{\text {buoy }} / D_{0}$, a more pronounced vortex ring structure exists even in the turbulent phase of the cloud evolution. This can be seen by comparing, for example, the vorticity component $\omega_{y}$ in the first and second sensitivity experiments $\left(\Delta T_{0}=0.8\right.$ and $1.8 \mathrm{~K}$, respectively, with $D_{0}=1000 \mathrm{~m}$ ) displayed in 

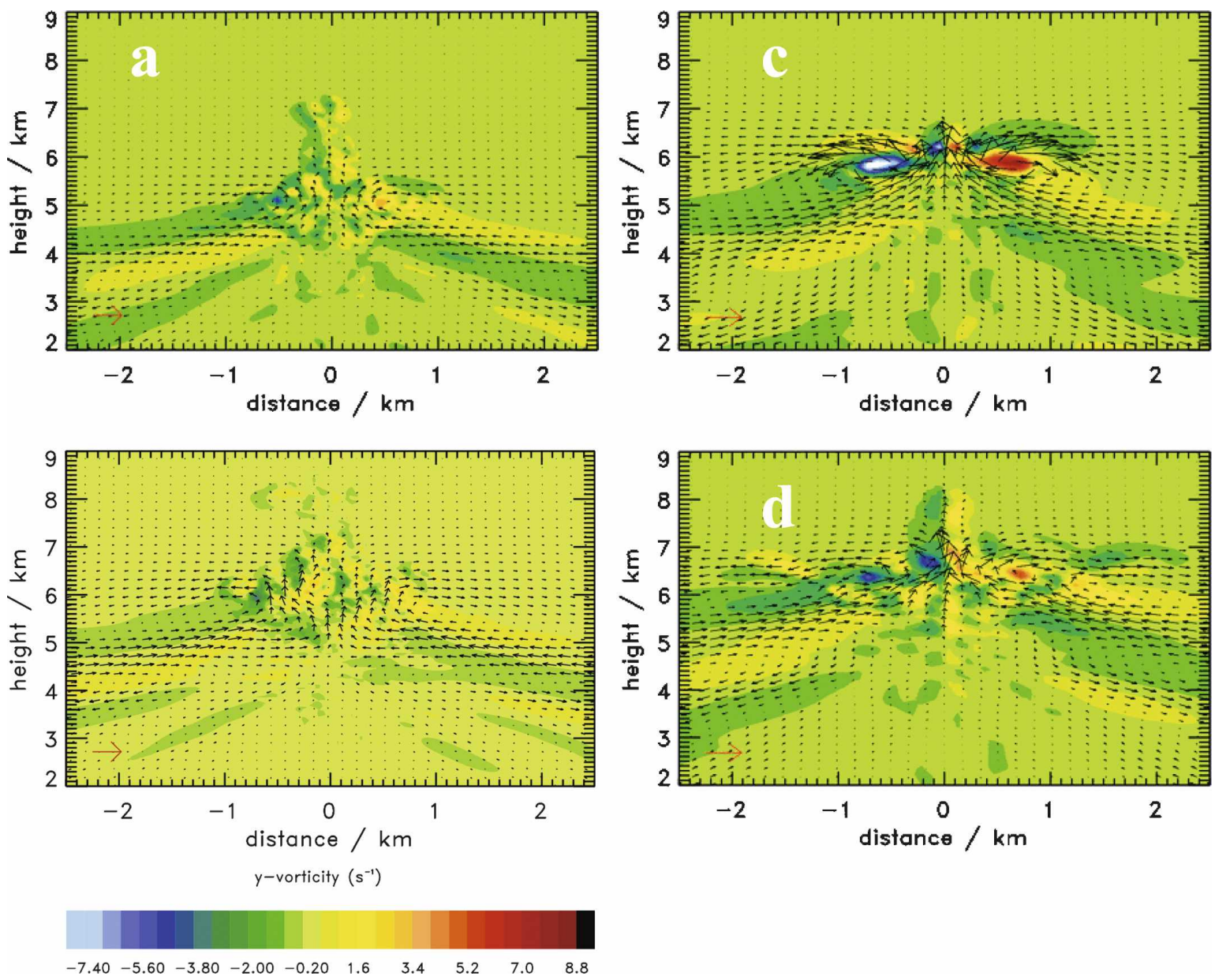

FIG. 6. Vertical cross sections of the $y$-averaged vorticity component $\omega_{y}$ (color shading) of the thermals (left) at $t=(\mathrm{a}) 24$ and (b) $36 \mathrm{~min}$ for $\Delta T_{0}=0.8 \mathrm{~K}$, and (right) at $t=$ (c) 16 and (d) $24 \mathrm{~min}$ for $\Delta T_{0}=1.8 \mathrm{~K}$. Panels (a) and (c) correspond to a nondimensional time of $t^{*}=7.4$, and (b) and (d) to $t^{*}=11.1$ (see text for details). Wind vectors are superimposed; red arrows mark the $0.5 \mathrm{~m} \mathrm{~s}{ }^{-1}$ magnitude.

Fig. 6. The results for the two experiments are shown for two nondimensional times close to and after the transition to the turbulent phase. Time is nondimensionalized using the buoyancy time scale $\tau=\left[L T_{\text {ref }} /\right.$ $\left.\left(g \Delta T_{0}\right)\right]^{1 / 2}$ (Stiller and Craig 2001).

Figure 7 summarizes the convergence results for the three sets of experiments. If the resolution required for convergence were determined by the initial thermal size $D_{0}$, then it would be independent of $\Delta T_{0}$ (as indicated by the dashed line in the figure, which corresponds to $\Delta x=0.15 D_{0}$ ). This is true for the second and third sensitivity experiments with $L_{\text {buoy }} / D_{0} \approx 0.60$ and 1.20 , respectively (displayed at the right-hand side of Fig. 7). The simulated thermals of both experiments qualitatively resemble a vortex ring, even in the turbulent phase. On the other hand, if $L_{\text {buoy }}$ is the important scale, the required resolution would change in proportion to $\Delta T_{0}$, as indicated by the solid line at $\Delta x=0.225$
$L_{\text {buoy }}$. This appears to be the case for the remaining experiments, with $L_{\text {buoy }} / D_{0} \approx 0.27,0.31$, and 0.33 , although the quantitative relationship between $\Delta x$ and $L_{\text {buoy }}$ is only approximately satisfied for the fourth experiment, indicating that the similarity revealed from the numerical simulations is not perfect.

\section{Conclusions}

This paper is motivated by the question of what spatial resolution is necessary to accurately simulate the transport, entrainment, and detrainment processes in evolving cumulus clouds. Analogous to many complex turbulent flows, it is hoped that LES will be possible; that is, there exists a dominant energy scale for the turbulent processes. Smaller-scale processes would then be controlled by a scale-invariant energy cascade, and accurate simulations would be obtained if the dom- 


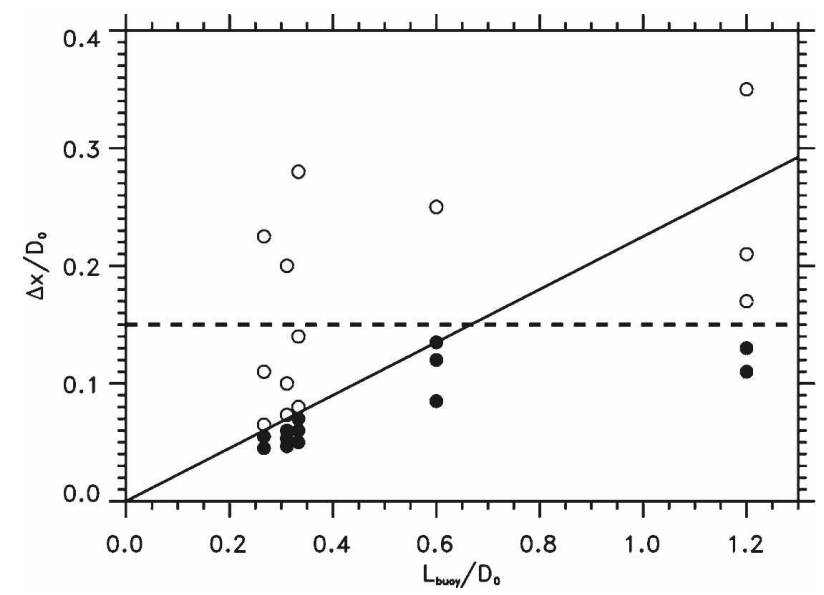

FIG. 7. Scatterplot showing the dimensionless resolution $\Delta x / D_{0}$ as a function of $L_{\text {buoy }} / D_{0}$ for all experiments, with open circles to indicate lack of convergence and solid dots for convergence according to the criterion defined in the text. The solid and dashed lines correspond to $\Delta x=0.225 L_{\text {buoy }}$ and $\Delta x=0.15 D_{0}$, respectively. The individual experiments can be identified by their values of $L_{\text {buoy }} / D_{0}$ and are, from left to right, the first and fourth sensitivity experiments, the control experiment, and the second and third sensitivity experiments (see text for details).

inant scale was resolved and the smaller scales were parameterized.

Based on theoretical arguments, two candidates for the dominant turbulence scale can be identified: the size of cloud $D_{0}$ (as for the classical plume evolution in a neutrally stratified environment; Morton et al. 1956) and the buoyancy scale $L_{\text {buoy }}=\Delta T_{0} / \Delta \Gamma$, which limits vertical displacements in an ambient thermal stratification (Stiller and Craig 2001). It is suggested that the smaller of these two scales should be resolved for LES of cumulus clouds. For atmospheric values this is likely to be $L_{\text {buoy, }}$ which is typically a few hundred meters.

Numerical simulations of a moist thermal were carried out for several values of $D_{0}$ and $L_{\text {buoy }}$ and with different spatial resolutions. The required resolution was identified by convergence of the radius-height curves for the simulated clouds. The radius-height curve depends on time-integrated entrainment and detrainment of the simulated thermal and is relatively insensitive to the particular realization of the turbulence in the individual numerical simulations. For experiments with various values of $L_{\text {buoy }}$ sufficiently small in comparison to $D_{0}$, the required resolution for convergence varied with $\Delta x \approx 0.2 L_{\text {buoy. }}$. For the two experiments with $D_{0}$ comparable or smaller than $L_{\text {buoy }}$, convergence was achieved for $\Delta x \approx 0.15 D_{0}$. These results are consistent with the hypothesis that the smaller of the two length scales determines the resolution requirement for LES of cumulus clouds.
Estimates for deep moist convection in the atmosphere given in the introduction suggest that $L_{\text {buoy }}$ is typically about an order of magnitude smaller than the size of the cumulus cloud as a whole. This implies that if an LES regime exists for cumulus convection, it will require a resolution of tens of meters, much finer than current cloud-permitting weather models with grid lengths on the order of $1 \mathrm{~km}$. Numerical models with this spatial resolution should not use a subgrid parameterization based on a large-eddy assumption, but rather a closure model that explicitly recognizes the unresolved turbulence scale. The same conclusion also holds for the simple entraining plume models used in cumulus parameterization schemes, which are almost universally based on the Morton et al. (1956) entrainment hypothesis that assumes scale-invariant turbulence on scales smaller than the thermal itself.

A significant limitation of the present study is the use of an idealized moist thermal. In particular, the lack of a turbulent subcloud layer results in an unrealistic laminar phase early in the simulations and a rising cloud base. Simulations of populations of clouds with $100-\mathrm{m}$ resolution are now possible (Khairoutdinov and Randall 2006; Grabowski et al. 2006), including a realistic, convectively modified boundary layer. Testing the convergence properties of such simulations would be an important next step, but even an analysis of a single such simulation might identify the relationship between buoyancy within a cloud and the scale of the turbulence prescribed by the buoyancy length scale.

The existence of a characteristic length scale for eddies in cumulus clouds that is smaller than the size of the cloud itself is consistent with observations that convective clouds are composed of several smaller thermals (see the review of Blyth 1993). For example, the dual-Doppler cloud radar analysis of Damiani et al. (2006) shows clear vortex ring structures in convective clouds, with core and tube diameters of 200-600 m, which is within the expected range of $L_{\text {buoy }}$ for typical cumulus buoyancies. Similar observations of large numbers of clouds, together with thermodynamic measurements, would provide the necessary basis to establish whether the buoyancy length was playing a significant role in cumulus mixing.

Acknowledgments. The authors appreciate the support of Romain Coharde and Anne Noeppel, who set up and performed parts of the numerical simulations during their training months at DLR. The EULAG computations were performed at the High Performance Computing Centers of the German Weather Service (DWD) in Offenbach and at the European Centre for Medium-Range Weather Forecasts (ECMWF). 


\section{APPENDIX}

\section{Determination of Cloud Parameters}

For dry simulations, a simulated thermal can be identified by a positive difference between the local potential temperature and its environmental value (i.e., by $\left.\Theta-\Theta_{e}>0\right)$. For moist simulations, the temperature excess is not appropriate to identify the turbulent cloud because motions of the environmental air in the stable background profile induce positive temperature anomalies outside the cloud as well. Accordingly, we defined the simulated cloud as the domain where the water vapor anomaly $q_{v}-q_{v e}>0.2 \mathrm{~g} \mathrm{~kg}^{-1}$. Because at a certain altitude $q_{u s}$ is smaller than this limiting value, a relative humidity $\mathrm{RH}>99 \%$ was the alternative criteria to define the cloud domain for heights above 7000 $\mathrm{m}$. Comparisons between the $q_{v}$ and $q_{c}$ fields showed that the above values resulted in a good definition of the cloud extent in terms of radius $R$, height $H$, and volume $V$ according to

$$
\begin{aligned}
& R=\frac{\sum_{i j k} \mathrm{RH}_{i j k} r_{i j}}{\sum_{i j k} \mathrm{RH}_{i j k}}, \quad H=\frac{\sum_{i j k} \mathrm{RH}_{i j k} z_{k}}{\sum_{i j k} \mathrm{RH}_{i j k}}, \quad \text { and } \\
& V=\sum_{i j k} d x_{i} d y_{j} d z_{k},
\end{aligned}
$$

where the indices $i, j$, and $k$ run over the whole threedimensional domain and $r_{i j}$ is the horizontal distance of the grid cell $(i, j)$ from the middle of the domain. Alternatively, we calculate $R_{\mathrm{Vol}}=(3 V / 4 \pi)^{(1 / 3)}$ as shown in Figs. $4 \mathrm{~d}$ and 5.

\section{REFERENCES}

Andrejczuk, M., W. W. Grabowski, S. P. Malinowski, and P. K. Smolarkiewicz, 2004: Numerical simulation of cloud-clear air interfacial mixing. J. Atmos. Sci., 61, 1726-1739.

,,--- , and,- 2006 : Numerical simulation of cloudclear air interfacial mixing: Effects on cloud microphysics. $J$. Atmos. Sci., 63, 3204-3225.

Bechtold, P., and Coauthors, 2000: A GCSS model intercomparison for a tropical squall line observed during TOGA COARE. II: Intercomparison of single-column models and a cloud-resolving model. Quart. J. Roy. Meteor. Soc., 126, 865888.

Blyth, A. M., 1993: Entrainment in cumulus clouds. J. Appl. Meteor., 32, 626-641.

Bretherton, C. S., 1997: Entrainment, detrainment, and mixing in atmospheric convection. The Physics and Parameterization of
Moist Atmospheric Convection, R. K. Smith, Ed., Kluwer, 211-230.

Bryan, G. H., J. C. Wyngaard, and J. M. Fritsch, 2003: Resolution requirements for the simulation of deep moist convection. Mon. Wea. Rev., 131, 2394-2416.

Carpenter, R. L., Jr., K. K. Droegemeier, and A. M. Blyth, 1998: Entrainment and detrainment in numerically simulated cumulus congestus clouds. Part I: General results. J. Atmos. Sci., 55, 3417-3422.

Damiani, R., G. Vali, and S. Haimov, 2006: The structure of thermals in cumulus from airborne dual-Doppler radar observations. J. Atmos. Sci., 63, 1432-1450.

Domaradzki, J. A., Z. Xiao, and P. K. Smolarkiewicz, 2003: Effective eddy viscosities in implicit large-eddy simulations of turbulent flows. Phys. Fluids, 15, 3890-3893.

Dörnbrack, A., J. D. Doyle, T. P. Lane, R. D. Sharman, and P. K. Smolarkiewicz, 2005: On physical realizability and uncertainty of numerical solutions. Atmos. Sci. Lett., 6, 118-122.

Emanuel, K. A., 1994: Atmospheric Convection. Oxford University Press, $580 \mathrm{pp}$.

Grabowski, W. W., and P. K. Smolarkiewicz, 1990: Monotone finite-difference approximations to the advection condensation problem. Mon. Wea. Rev., 118, 2082-2097.

, and —, 2002: A multiscale anelastic model for meteorological research. Mon. Wea. Rev., 130, 939-956.

_ land: A model intercomparison based on LBA observations. Quart. J. Roy. Meteor. Soc., 132, 317-344.

Guichard, F., and Coauthors, 2004: Modelling the diurnal cycle of deep precipitating convection over land with cloud-resolving models and single-column models. Quart. J. Roy. Meteor. Soc., 130, 3139-3172.

Houze, R. A., Jr., 1993: Cloud Dynamics. Academic Press, 570 pp.

Khairoutdinov, M., and D. Randall, 2006: High-resolution simulation of shallow-to-deep convection transition over land. $J$. Atmos. Sci., 63, 3421-3436.

Morton, B. R., G. I. Taylor, and J. S. Turner, 1956: Turbulent gravitational convection from maintained and instantaneous sources. Proc. Roy. Soc. London, 234A, 1-23.

Reveley, J., 2005: University of Reading weather-photography competition, 2004. Weather, 60, 143.

Smolarkiewicz, P. K., and L. G. Margolin, 1997: On forward-intime differencing for fluids: An Eulerian/semi-Lagrangian non-hydrostatic model for stratified flows. Atmos.-Ocean, 35, $127-152$

_, and - 1998: MPDATA: A finite-difference solver for geophysical flows. J. Comput. Phys., 140, 459-480.

_ physical turbulence: Continuous mapping approach. Int. J. Numer. Methods Fluids, 47, 789-801.

— L. G. Margolin, and A. A. Wyszogrodzki, 2001: A class of nonhydrostatic global models. J. Atmos. Sci., 58, 349-364.

Stiller, O., and G. C. Craig, 2001: A scaling hypothesis for moist convective updraughts. Quart. J. Roy. Meteor. Soc., 127, 1551-1570.

Wyngaard, J. C., 2004: Toward numerical modeling in the "Terra Incognita.” J. Atmos. Sci., 61, 1816-1826. 